\title{
The Phase of Ongoing Oscillations Mediates the Causal Relation between Brain Excitation and Visual Perception
}

\author{
Laura Dugué, ${ }^{1,2}$ Philippe Marque, ${ }^{3}$ and Rufin VanRullen ${ }^{1,2}$ \\ ${ }^{1}$ CNRS, UMR5549, centre de recherche Cerveau et Cognition, Faculté de Médecine de Purpan, 31062 Toulouse, France, ${ }^{2}$ Université Paul Sabatier, 31062 \\ Toulouse, France, and ${ }^{3}$ Médecine Physique et de réadaptation, CHU Rangueil, 31062 Toulouse, France
}

Why does neuronal activity in sensory brain areas sometimes give rise to perception, and sometimes not? Although neuronal noise is often invoked as the key factor, a portion of this variability could also be due to the history and current state of the brain affecting cortical excitability. Here we directly test this idea by examining whether the phase of prestimulus oscillatory activity is causally linked with modulations of cortical excitability and with visual perception. Transcranial magnetic stimulation (TMS) was applied over human visual cortex to induce illusory perceptions (phosphenes) while electroencephalograms (EEGs) were simultaneously recorded. Subjects reported the presence or absence of an induced phosphene following a single pulse of TMS at perceptual threshold. The phase of ongoing alpha $(\sim 10 \mathrm{~Hz})$ oscillations within $400 \mathrm{~ms}$ before the pulse significantly covaried with the perceptual outcome. This effect was observed in occipital regions around the site of TMS, as well as in a distant frontocentral region. In both regions, we found a systematic relationship between prepulse EEG phase and perceptual performance: phosphene probability changed by 15\% between opposite phases. In summary, we provide direct evidence for a chain of causal relations between the phase of ongoing oscillations, neuronal excitability, and visual perception: ongoing oscillations create periodic "windows of excitability," with sensory perception being more likely to occur at specific phases.

\section{Introduction}

A growing body of experiments attempt to explain the perceptual variability observed for successive presentations of a given stimulus in apparently unchanging experimental conditions. In particular, it is now well known that the amplitude of prestimulus oscillations in the alpha range $(\sim 10 \mathrm{~Hz})$ significantly affects the perceptual outcome (Ergenoglu et al., 2004; Hanslmayr et al., 2007; Kanai et al., 2008; van Dijk et al., 2008; Romei et al., 2010). Alpha power over posterior cortical regions also predicts cortical excitability, since it modulates the probability that a transcranial magnetic stimulation (TMS) pulse will elicit the perception of an illusory flash or "phosphene" (Romei et al., 2008). Ongoing activity is not only characterized by its amplitude, however, but also by its instantaneous phase. This dynamic variable has been related to the probability of neuronal firing (Lakatos et al., 2005; VanRullen et al., 2005; Fries et al., 2007; Montemurro et al., 2008; Whittingstall and Logothetis, 2009) and was recently found to affect the probability of perceiving a weak visual stimulus (Busch et al., 2009; Mathewson et al., 2009). In this study, we sought to establish a direct causal link between EEG phase, cortical excitability, and visual perception. To do so, we applied single pulses of TMS over the occipital region while we simultaneously re-

\footnotetext{
Received March 7, 2011; revised June 6, 2011; accepted June 26, 2011.

Author contributions: L.D. and R.V. designed research; L.D. and P.M. performed research; L.D. and R.V. analyzed data; L.D. and R.V. wrote the paper.

This research was funded by a EURYI Award and an Agence Nationale pour la Recherche Grant 06JCJC-0154 to R.V.

Correspondence should be addressed to either Laura Dugué or Rufin VanRullen at the above addresses, E-mail: laura.dugue@cerco.ups-tlse.fr or rufin.vanrullen@cerco.ups-tlse.fr.

DOI:10.1523/JNEUROSCI.1161-11.2011

Copyright $\odot 2011$ the authors $\quad 0270-6474 / 11 / 3111889-05 \$ 15.00 / 0$
}

corded EEG. We measured the efficiency of the pulse at inducing a perceptual phosphene (corresponding to our definition of cortical excitability) as a function of the prepulse oscillatory phase, and found a strong relation between cortical excitability and the phase of ongoing alpha oscillations.

\section{Materials and Methods}

\section{Participants}

Seventeen subjects ( 7 females), aged 20-35, enrolled in the experiments. Four were not included because they were not able to see a TMS-induced phosphene. Of the 13 subjects that participated, one was excluded from analysis because the TMS pulses produced EEG artifacts that did not recover within $200 \mathrm{~ms}$; three were excluded from analysis because no significant ERP difference was found between phosphene and nophosphene trials (see below). In the end, data from nine subjects were analyzed. All participants gave written informed consent before taking part in the experiment. Standard exclusion criteria for TMS were applied (Wassermann, 1998; Rossi et al., 2009). The study was approved by the local ethics committee "Sud-Ouest et Outre-Mer I" (protocol number 2009-A01087-50).

\section{Procedure}

TMS parameters characterization. Participants were first tested on their ability to perceive a phosphene (an illusory flash produced by the magnetic stimulation of the occipital cortex) at suprathreshold intensity: we used a train of 7 pulses at $20 \mathrm{~Hz}$ and at $70 \%$ of TMS machine output intensity applied over the right occipital cortex. Four of 17 subjects were excluded at this stage because of their inability to perceive a phosphene. In a second step, single TMS pulses were applied at varying intensities. For each trial, subjects indicated whether they had perceived a phosphene or not. This procedure allowed us to determine the individual phosphene perception intensity threshold, which was then used in the experimental session. 

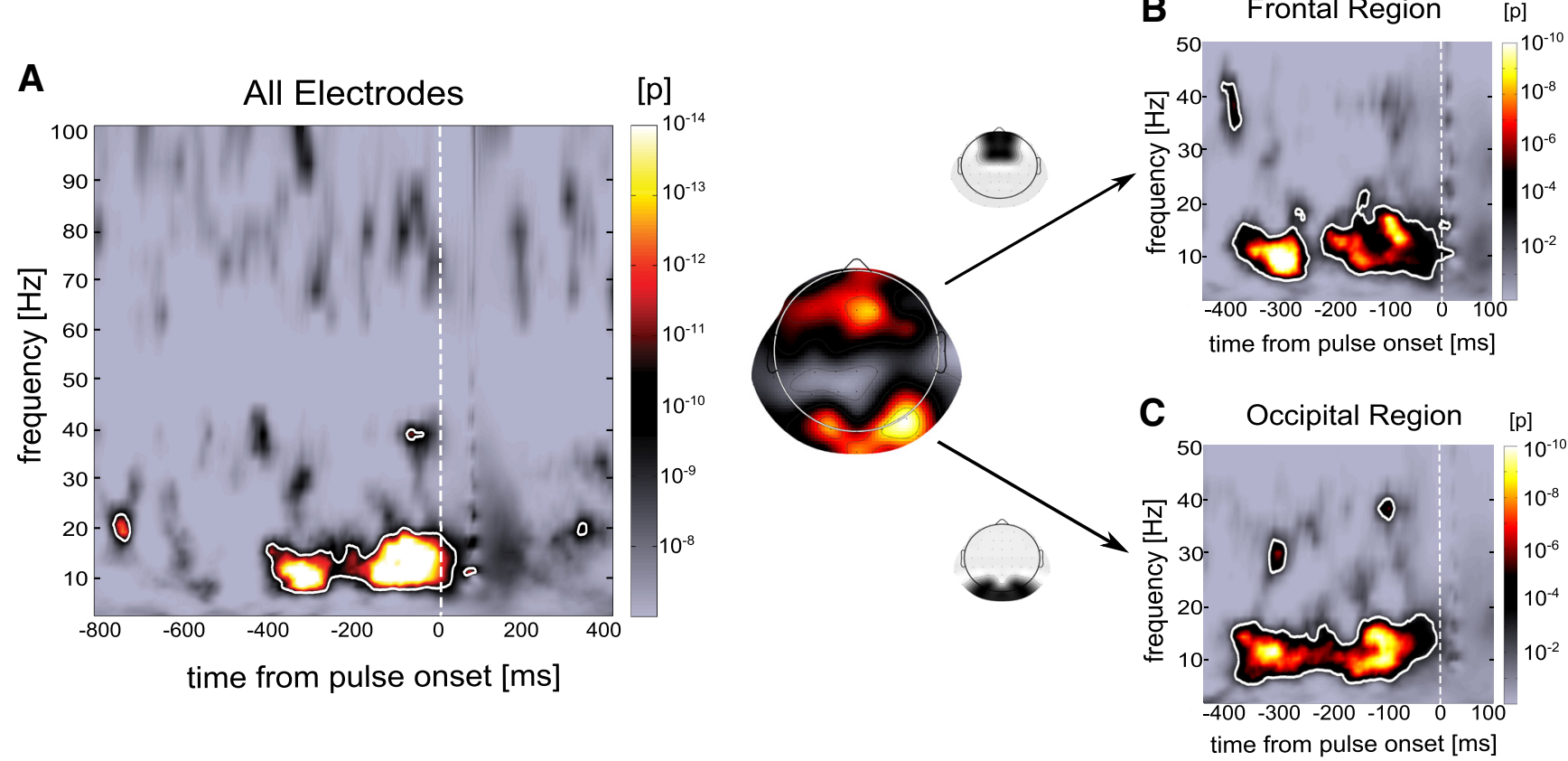

Figure 1. Prepulse phase predicts phosphene perception. $A$, Statistical comparison between phase-locking values for trials grouped according to perception (phosphene/nophosphene, averaged over 64 electrodes and 9 subjects) and surrogate phase-locking values computed over random subsets of trials. A significant effect indicates that ongoing phase differs statistically for phosphene and no-phosphene trials. The color map represents uncorrected $p$ values (similar in $B$ and $C$ ) and the white outline indicates significant effects corrected for multiple comparisons using the FDR method. There is a strongly significant phase effect (FDR $=10^{-5}$; corresponding to a $p$ value threshold of $6.4 \times 10^{-7}$ ) in the last $400 \mathrm{~ms}$ preceding the pulse, ranging in frequency from 7 to $17 \mathrm{~Hz}$. The topography shows the scalp distribution of phase-locking values in the time-frequency range of 7 to $17 \mathrm{~Hz}$ and -400 to $-50 \mathrm{~ms}$. Further analyses are performed on two regions of interest: a frontal and an occipital one. $\boldsymbol{B}$, Statistical significance of the phase difference between phosphene and no-phosphene trials, in the frontal region of interest at each time-frequency point. There is a strongly significant phase effect ( $F D R=10^{-3}$; corresponding to a $p$ value threshold of $\left.6.2 \times 10^{-5}\right)$ in the $400 \mathrm{~ms}$ preceding the pulse and in the alpha frequency range $(\sim 10 \mathrm{~Hz})$. C, Same as $\boldsymbol{B}$, but for the occipital region of interest.

Experimental session. Each participant performed four blocks of 200 trials. In $90 \%$ of the trials, they received a single TMS pulse at the previously determined threshold intensity, while in the last $10 \%$ (randomly interleaved) two successive pulses of the same intensity were applied, separated by $40 \mathrm{~ms}$. These "catch" trials were intended to monitor the validity of subjective responses: with a double pulse, we expected significantly more phosphene trials. Participants were seated $57 \mathrm{~cm}$ from a dark screen $\left(36.5^{\circ} \times 27^{\circ}\right.$ of visual angle $)$ and were instructed to fixate a central dot. Each trial began when the subject pressed a button on the keyboard. The interval between the button press and the subsequent TMS pulse varied randomly between 1.5 and $2.5 \mathrm{~s}$ (uniform distribution). A response screen appeared $0.6 \mathrm{~s}$ after the pulse, and subjects indicated whether they had perceived a phosphene (left keyboard arrow) or not (right arrow). To avoid any kind of habituation, practice effect or change in arousal affecting the phosphene intensity threshold, when subjects obtained $>75 \%$ or $<25 \%$ of phosphene trials in a running window of 20 trials, the block was paused and the experimenter remeasured the threshold and adjusted the intensity accordingly, so as to maintain a ratio of $\sim 50 \%$ of phosphene trials.

\section{TMS apparatus and EEG acquisition}

Participants' head was maintained using a chinrest and a headrest in front of them and a $70 \mathrm{~mm}$ figure-of-eight coil was pressed against their scalp in the right occipital region, leading to a stable position. TMS was applied using a Magstim Rapid ${ }^{2}$ stimulator of 3.5 tesla, which produces a biphasic current. EEG was acquired continuously with a 64-channels ActiveTwo Biosemi system. Two additional electrodes, CMS (Common Mode Sense) and DRL (Driven Right Leg), were used as reference and ground. These electrodes were placed on the subjects' face $2 \mathrm{~cm}$ under the eyes (CMS on the left and DRL on the right) to minimize TMSinduced EEG artifacts. Horizontal and vertical electro-oculograms were recorded by three additional electrodes around the subjects' eyes. Data were downsampled to $512 \mathrm{~Hz}$, re-referenced to average reference, and epoched from $-1500 \mathrm{~ms}$ before the pulse to $1000 \mathrm{~ms}$ after the pulse. Finally, EEG data immediately following the pulse ( - 1 $\mathrm{ms}$ before the pulse to $150 \mathrm{~ms}$ after the pulse) was erased and replaced with a linear interpolation of the window boundaries, so as to minimize the pulse-induced artifacts. (We verified that the slope of this linear interpolation was unrelated to the perceptual outcome: the comparison across subjects of the averaged slopes between phosphene and no-phosphene trials was not significant, $t_{(8)}=1.30, p=0.23$ ). Manual artifact rejection was further performed on the data. One subject was excluded from further analysis because the EEG recordings took $>200 \mathrm{~ms}$ after each pulse to recover from the TMS artifacts, and the linear interpolation procedure was thus inefficient.

\section{EEG analysis}

Event-related potentials were computed as the average of all the trials for each condition (phosphene trials and no-phosphene trials). For this analysis, a notch filter at $50 \mathrm{~Hz}$ was applied to remove power line artifacts. The difference of ERPs between the two conditions was plotted for electrode PO3 for each subject: based on the results of Taylor et al. (2010) (Taylor et al., 2010), we expected higher amplitudes in the phosphene trials after $300 \mathrm{~ms}$ after the pulse. We used this test to confirm that subjective reports depended on a genuine perceptual difference between the two trial conditions. Three subjects were excluded at this stage of the analysis, because the difference did not reach $1.5 \mu \mathrm{V}$ in amplitude. Phase information was then computed using a time-frequency transform (akin to wavelets) on single trials using the eeglab software package under Matlab (function timefreq with parameters "cycles" and "freqs" set to $[1,15]$ and $[2,100]$, respectively). These parameters produce frequencies that increase linearly from 2 to $100 \mathrm{~Hz}$, while the length of the filter increases linearly from 1 to 15 cycles. We also performed the same analysis with one-cycle wavelets at all frequencies, with comparable results (data not shown). We calculated the prepulse phase locking (the extent of phase concentration across trials) at each frequency and time point separately for each condition (phosphene trials and no-phosphene trials). The average of these two values was compared to a surrogate distribution 
A

Difference: Phosphene vs. No Phosphene (electrode PO3)

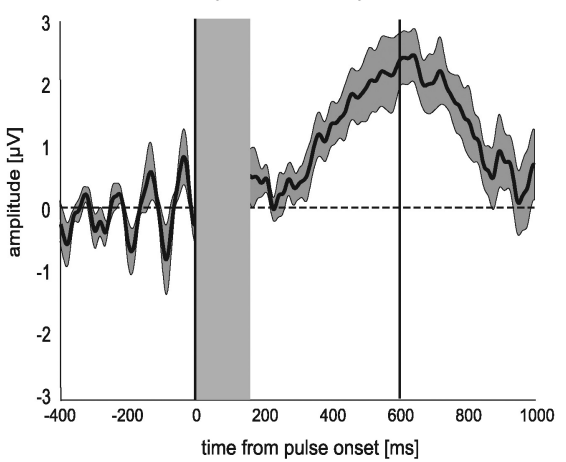

C

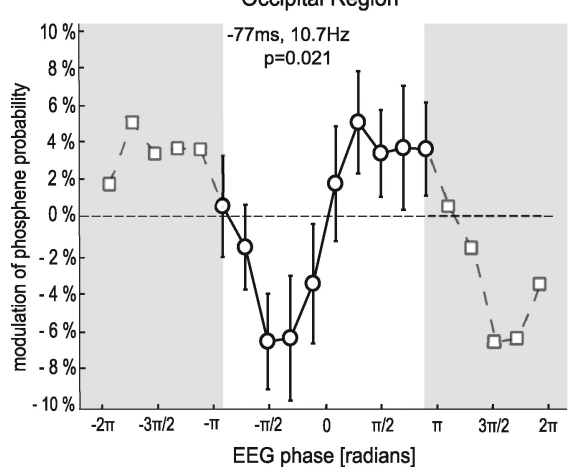

B

Difference: Phosphene vs. No Phosphene (electrode AFz)

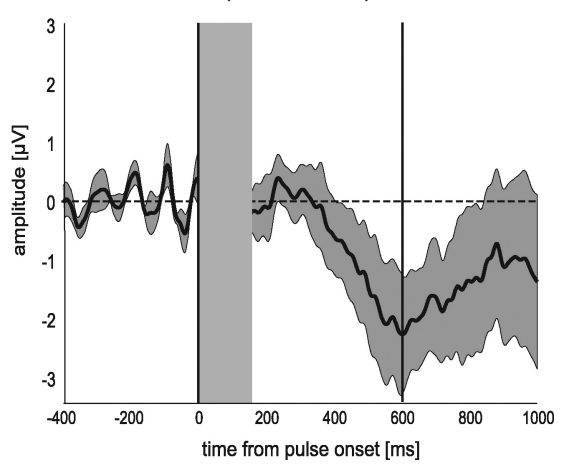

D

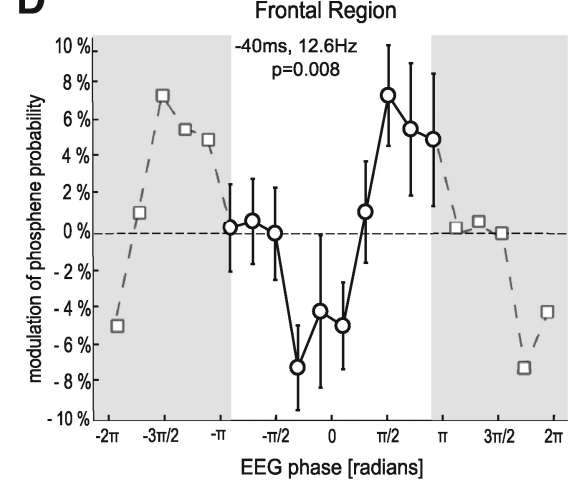

Figure 2. Differential activity (top), and periodic fluctuations of phosphene probability (bottom). $\boldsymbol{A}$, Difference of ERPs between phosphene trials and no-phosphene trials for an occipital electrode (P03). $\boldsymbol{B}$, Difference of ERPs for a frontal electrode (AFz). The shaded area around the ERP difference corresponds to the SEM across nine subjects (SEM). The gray rectangle from $-1 \mathrm{~ms}$ to $150 \mathrm{~ms}$ masks the duration of electrical artifacts induced by the TMS pulse. The black line at $0 \mathrm{~ms}$ corresponds to the pulse onset and at 600 ms to the onset of the response screen, asking subjects to report the presence or absence of a phosphene. The ERP difference diverges from zero between 250 and $600 \mathrm{~ms}$ after the pulse, supporting the notion that the two conditions differ at the perceptual level. In addition, the difference oscillates during the 400 ms before the pulse onset, suggesting that the two conditions correspond to different prepulse phases. $\boldsymbol{C}$, Relationship between the prepulse occipital EEG phase (expressed in radians) at $-77 \mathrm{~ms}$ and $10.7 \mathrm{~Hz}$ (time-frequency point of maximal significance), and phosphene perception (expressed as a modulation of average phosphene probability). Single trials were sorted in 10 phase bins. Phosphene probability was computed for each phase bin, then averaged over all electrodes in the occipital region of interest and over all subjects (error bars represent SEM across subjects). For enhanced readability, the curve is plotted over two consecutive cycles (phase values that were replicated are represented over shaded backgrounds). The curve demonstrates that phosphene report probability significantly oscillates along with ongoing EEG phase (one-way ANOVA over the 10 phase values, $F_{(9,89)}=2.35, p=0.021$ ). The magnitude of this periodic modulation (measured between optimal and opposite phase values) is $\sim 13 \%$. D, Relationship between the prepulse EEG phase at $-40 \mathrm{~ms}$ and $12.6 \mathrm{~Hz}$ (time-frequency point of maximal significance) and phosphene perception, averaged over all electrodes of the frontal region of interest. Plotting conventions are similar to those in C. As previously, phosphene probability significantly oscillates along with ongoing EEG phase (one-way ANOVA, $F_{(9,89)}=2.72, p=0.008$ ). The magnitude of this periodic modulation is $\sim 15 \%$.

(bootstrap procedure): the experimental trials were randomly assigned to one of the two conditions (keeping the number of trials in each condition constant), and phase lockings were recomputed; by repeating this procedure 10,000 times, we determined a distribution of phase-locking values under the null hypothesis (i.e., that there is no systematic phase difference between phosphene and no-phosphene trials). This distribution was characterized by its mean and SD, which were later combined across subjects and electrodes. Finally, the experimentally observed phase locking at each time and frequency point was turned into a $p$ value ( $t$ test) by comparing it to the mean and SD of the corresponding null distribution (Fig. 1). The false discovery rate (FDR) method was used to correct for multiple comparisons to avoid type I errors $\left(\mathrm{FDR}=10^{-5}\right.$, corresponding to a $p$ value threshold of $6.4 \times 10^{-7}$ ). To ensure that the results were not due to a single subject (outlier), we removed each subject one by one and recomputed the $p$ values and the FDR (FDR $=0.05$, corresponding to $p$ value thresholds $<0.0017)$. After a topographical analysis revealed the regions that participated in the phase-locking effect, we repeated the previous analysis for each region of interest. An FDR procedure $\left(\mathrm{FDR}=10^{-3}\right.$, corresponding to a $p$ value threshold of $4.8 \times 10^{-5}$ for the occipital ROI and $6.2 \times 10^{-5}$ for the frontal ROI) was also applied, and we ensured as previously that the obtained effects were not only the consequence of any single subject.

\section{Results}

In $90 \%$ of the 800 experimental trials, we applied single-pulse TMS over the right occipital region of 9 healthy subjects at threshold intensity. For these trials, subjects reported perceiving a phosphene with $45.96 \%$ probability ( $\pm 7.68 \%$ SEM). In the remaining $10 \%$ of the trials (randomly interleaved), we applied doublepulse TMS at the same intensity of stimulation. These "catch trials" served to control the accuracy of the subjective reports of phosphene perception. Indeed, the probability of reporting a phosphene after these double pulses increased to $91.66 \%( \pm 8.81 \%)$.

Simultaneously with magnetic stimulation, we recorded ongoing brain activity using EEG. We first focused on the prepulse time window and tested whether ongoing oscillatory phase differed between the trials in which the single pulse was successful versus unsuccessful in eliciting a phosphene. To this end, we computed the phase locking (the extent of phase concentration across trials; see Materials and Methods) separately for the two groups of trials (phosphene/no phosphene) and compared the values statistically (bootstrap procedure, see Materials and Methods) with phase-locking values computed on the same numbers of trials, but randomly chosen regardless of the perceptual outcome. When averaged over the 9 subjects and 64 electrodes, this measure revealed a strong phase difference between phosphene and no-phosphene conditions $\left(p=10^{-14}\right)$ in the last $400 \mathrm{~ms}$ before the pulse, between 7 and $17 \mathrm{~Hz}$ (Fig. $1 A$ ). The effect remained significant after correction for multiple comparisons across time and frequencies using the FDR procedure (FDR $=10^{-5}$, corresponding to a $p$ value threshold of $\left.6.4 \times 10^{-7}\right)$. We also verified that the result was not due to any single subject, by removing each subject one by one from our pool and recomputing our statistical measures: each time a significant effect persisted within the same time-frequency region.

The topography of phase-locking values in the time-frequency window $-400 \mathrm{~ms}$ to $-50 \mathrm{~ms}$ relative to the pulse and 7 to $17 \mathrm{~Hz}$ indicated that the previously observed effect was mainly due to two regions of interest: an occipital and a frontocentral region (Fig. 1). Consequently, we recomputed the phase-locking values for these two regions (Fig. $1 B, C$ ). In both cases, we again 
observed a strong phase difference within $400 \mathrm{~ms}$ before the pulse and in the alpha band $(\sim 10 \mathrm{~Hz})$. As before, we verified that these results were not merely caused by a single subject.

To further understand the relation between prestimulus ongoing oscillations and poststimulus cortical activity, for both regions we chose one representative electrode [PO3, based on previous results by Taylor et al. (2010); AFz, a central frontal electrode] and computed the difference of ERPs between the "phosphene" trials and the "no-phosphene" trials (Fig. 2A,B). Both electrodes showed significant differential activity, starting at $\sim 300 \mathrm{~ms}$ and peaking at $\sim 600 \mathrm{~ms}$ after the pulse (positive in the case of $\mathrm{PO} 3$, in accordance with previous data (Taylor et al., 2010); negative in the case of $\mathrm{AFz}$ - the existence of such a significant postpulse ERP difference was taken as an indicator of veridical phosphene perception, and used to select subjects for all EEG analyses, as detailed in the Materials and Methods section). Interestingly, in both cases there was a clear oscillation of the differential ERP in the last $400 \mathrm{~ms}$ preceding the pulse, with a periodicity of $\sim 100 \mathrm{~ms}$. This finding corroborates the results presented in Figure 1: if perceiving versus not perceiving a phosphene depends on two opposite prestimulus alpha phases, then it makes sense that the direct subtraction of ERPs for these two conditions should oscillate in the alpha range.

Finally, to quantify the relation between prestimulus alpha phase and cortical excitability, we sorted the single trials in 10 phase bins for each electrode of the two regions of interest at the time-frequency point of maximal significance (Fig. 2C,D), and for each bin we determined the phosphene report probability. More precisely, phosphene probability was computed for each phase bin, then averaged over all electrodes for each region of interest and over all subjects. In both regions, phosphene perception was systematically and significantly modulated by alpha phase (one-way ANOVA, $F_{(9,89)}=2.35, p=0.021$ for the occipital region; $F_{(9,89)}=2.72, p=0.008$ for the frontal region). In fact, between the optimal phase angle and the opposite one, phosphene perception varied by as much as $15 \%$ on average.

\section{Discussion}

The key finding of this study is that the causal relation between cortical excitation and phosphene perception depends on $\sim 10$ $\mathrm{Hz}$ ongoing EEG phase. Recent studies highlighted the importance of $\sim 10 \mathrm{~Hz}$ ongoing oscillatory phase in visual perception (Busch et al., 2009; Mathewson et al., 2009; Busch and VanRullen, 2010; Drewes and VanRullen, 2011). For the first time, we directly establish the chain of causal events linking the phase of the ongoing EEG signal recorded just before the stimulation, the excitability of the sensory cortex at the moment of stimulation, and the conscious perception that ensues. By using direct cortical stimulation rather than external sensory stimuli, we were also able to identify the oscillatory phase values that promote or impede cortical excitability: Figure 2 indicates that cortical activation occurring between the peak (phase $=0$ ) and the next zero-crossing (phase $=\pi / 2$ ) of the occipital EEG alpha oscillation is most likely to produce a perceptual outcome (these values were derived by observing the waveform in Fig. $2 \mathrm{~A}$, as well as the phase effect at $-77 \mathrm{~ms}$ and $10.7 \mathrm{~Hz}$ in Fig. 2C - extrapolated to the time of stimulation). Our results suggest that oscillations of ongoing activity reflect cyclic variations of cortical excitability (Bishop, 1933; Lindsley, 1952; Harter, 1967; Buzsáki and Draguhn, 2004; Vanrullen et al., 2005; Fries et al., 2007; Rajkai et al., 2008; Sirota et al., 2008), supporting the concept of "perceptual moments," i.e., the notion that perception could rapidly oscillate between favorable and less favorable periods (Harter, 1967;
Stroud, 1967; Varela et al., 1981; Purves et al., 1996; VanRullen and Koch, 2003; Smith et al., 2006).

Another critical result is that two distinct areas (one occipital and one frontocentral) displayed a significant relation between EEG phase and phosphene perception. Previous studies had linked ongoing phase recorded within a very similar frontocentral region to the probability of consciously perceiving a brief flash of light (Busch et al., 2009), and further demonstrated that this phasic influence actually mirrored the periodic fluctuations of attention (Busch and VanRullen, 2010). In this context, one could speculate that $\sim 10 \mathrm{~Hz}$ oscillations over occipital cortex cyclically modulate the buildup of a sensory percept, while concurrent oscillations in frontocentral regions, under the control of attention, periodically facilitate the conscious report of this percept. Future work could be directed at understanding the interplay between these two sources of periodic fluctuations in visual perception.

\section{References}

Bishop G (1933) Cyclic changes in excitability of the optic pathway of the rabbit. Am J Physiol 103:213-224.

Busch NA, VanRullen R (2010) Spontaneous EEG oscillations reveal periodic sampling of visual attention. Proc Natl Acad Sci U S A 107:16048-16053.

Busch NA, Dubois J, VanRullen R (2009) The phase of ongoing EEG oscillations predicts visual perception. J Neurosci 29:7869-7876.

Buzsáki G, Draguhn A (2004) Neuronal oscillations in cortical networks. Science 304:1926-1929.

Drewes J, VanRullen R (2011) This is the rhythm of your eyes: the phase of ongoing electroencephalogram oscillations modulates saccadic reaction time. J Neurosci 31:4698-4708.

Ergenoglu T, Demiralp T, Bayraktaroglu Z, Ergen M, Beydagi H, Uresin Y (2004) Alpha rhythm of the EEG modulates visual detection performance in humans. Brain Res Cogn Brain Res 20:376-383.

Fries P, Nikolić D, Singer W (2007) The gamma cycle. Trends Neurosci 30:309-316.

Hanslmayr S, Aslan A, Staudigl T, Klimesch W, Herrmann CS, Bäuml KH (2007) Prestimulus oscillations predict visual perception performance between and within subjects. Neuroimage 37:1465-1473.

Harter MR (1967) Excitability cycles and cortical scanning: a review of two hypotheses of central intermittency in perception. Psychol Bull 68:47-58.

Kanai R, Chaieb L, Antal A, Walsh V, Paulus W (2008) Frequency-dependent electrical stimulation of the visual cortex. Curr Biol 18:1839-1843.

Lakatos P, Shah AS, Knuth KH, Ulbert I, Karmos G, Schroeder CE (2005) An oscillatory hierarchy controlling neuronal excitability and stimulus processing in the auditory cortex. J Neurophysiol 94:1904-1911.

Lindsley DB (1952) Psychological phenomena and the electroencephalogram. Electroencephalogr Clin Neurophysiol 4:443-456.

Mathewson KE, Gratton G, Fabiani M, Beck DM, Ro T (2009) To see or not to see: prestimulus $\alpha$ phase predicts visual awareness. J Neurosci 29:2725-2732.

Montemurro MA, Rasch MJ, Murayama Y, Logothetis NK, Panzeri S (2008) Phase-of-firing coding of natural visual stimuli in primary visual cortex. Curr Biol 18:375-380.

Purves D, Paydarfar JA, Andrews TJ (1996) The wagon wheel illusion in movies and reality. Proc Natl Acad Sci U S A 93:3693-3697.

Rajkai C, Lakatos P, Chen CM, Pincze Z, Karmos G, Schroeder CE (2008) Transient cortical excitation at the onset of visual fixation. Cereb Cortex 18:200-209.

Romei V, Brodbeck V, Michel C, Amedi A, Pascual-Leone A, Thut G (2008) Spontaneous fluctuations in posterior alpha-band EEG activity reflect variability in excitability of human visual areas. Cereb Cortex 18:2010-2018.

Romei V, Gross J, Thut G (2010) On the role of prestimulus alpha rhythms over occipito-parietal areas in visual input regulation: correlation or causation? J Neurosci 30:8692-8697.

Rossi S, Hallett M, Rossini PM, Pascual-Leone A (2009) Safety, ethical considerations, and application guidelines for the use of transcranial magnetic stimulation in clinical practice and research. Clin Neurophysiol 120:2008-2039.

Sirota A, Montgomery S, Fujisawa S, Isomura Y, Zugaro M, Buzsáki G (2008) Entrainment of neocortical neurons and gamma oscillations by the hippocampal theta rhythm. Neuron 60:683-697. 
Smith ML, Gosselin F, Schyns PG (2006) Perceptual moments of conscious visual experience inferred from oscillatory brain activity. Proc Natl Acad Sci U S A 103:5626-5631.

Stroud JM (1967) The fine structure of psychological time. Ann N Y Acad Sci 138:623-631.

Taylor PC, Walsh V, Eimer M (2010) The neural signature of phosphene perception. Hum Brain Mapp 31:1408-1417.

van Dijk H, Schoffelen JM, Oostenveld R, Jensen O (2008) Prestimulus oscillatory activity in the alpha band predicts visual discrimination ability. J Neurosci 28:1816-1823.

VanRullen R, Koch C (2003) Is perception discrete or continuous? Trends Cogn Sci 7:207-213.
VanRullen R, Guyonneau R, Thorpe SJ (2005) Spike times make sense. Trends Neurosci 28:1-4.

Varela FJ, Toro A, John ER, Schwartz EL (1981) Perceptual framing and cortical alpha rhythm. Neuropsychologia 19:675-686.

Wassermann EM (1998) Risk and safety of repetitive transcranial magnetic stimulation: report and suggested guidelines from the International Workshop on the Safety of Repetitive Transcranial Magnetic Stimulation, June 5-7, 1996. Electroencephalogr Clin Neurophysiol 108:1-16.

Whittingstall K, Logothetis NK (2009) Frequency-band coupling in surface EEG reflects spiking activity in monkey visual cortex. Neuron 64:281-289. 